\title{
Atuação do sanitarista em município brasileiro de tríplice-fronteira: Covid-19 como emergência de saúde coletiva
}

\author{
Hygienist's practice in a triple-border Brazilian \\ municipality: Covid-19 as a collective health \\ emergency
}

Roberth Steven Gutiérrez Murillo', Olga Lucía Mosquera Conde², Gustavo Cezar Wagner Leandro ${ }^{3}$, Hannalícia Bueno de Freitas ${ }^{4}$, Erica Ferreira de Souza ${ }^{5}$

1. ORCID: http://orcid.org/0000-0003-2304-3241. Sanitarista Residente no Programa de Residência Multiprofissional em Saúde da Família. Universidade Federal da Integração Latino-americana, Foz do Iguaçu, Paraná, Brasil. E-mail: stevengumu@gmail.com.

2. ORCID: http://orcid.org/0000-0002-4988-1764. Sanitarista Residente no Programa de Residência Multiprofissional em Saúde da Família. Universidade Federal da Integração Latino-americana, Foz do Iguaçu, Paraná, Brasil. E-mail: olgalum09@gmail.com.

3. ORCID: http://orcid.org/0000-0002-5014-2387. Sanitarista Residente no Programa de Residência Multiprofissional em Saúde da Família. Universidade Federal da Integração Latino-americana, Foz do Iguaçu, Paraná, Brasil. E-mail: gustavocesar_leandro@hotmail.com.

4. ORCID: http://orcid.org/0000-0001-5110-1424. Sanitarista Residente no Programa de Residência Multiprofissional em Saúde da Família. Universidade Federal da Integração Latino-americana, Foz do Iguaçu, Paraná, Brasil. E-mail: hannabfreitas@gmail.com.

5. ORCID: http://orcid.org/0000-0002-5425-145X. Enfermeira Mestre em Saúde Pública Baseada em Evidências. Universidade Federal de Pelotas, Pelotas, Rio Grande do Sul, Brasil. E-mail: ericafesil@hotmail.com.

CONTATO: Roberth Steven Gutiérrez Murillo | Endereço: Avenida Brodoski 1095, Jardim Santa Rosa, Vila A, Foz do Iguaçu - Paraná Telefone: (45) 98840-6824 | E-mail: stevengumu@gmail.com

RESUMO Em virtude da atual crise sociossanitária decorrente da nova manifestação do coronavírus, este relato de experiência buscou descrever a inserção do profissional sanitarista no Departamento de Vigilância Epidemiológica de Foz do Iguaçu/PR, no âmbito da prevenção e combate à Covid-19 em território brasileiro de tríplice- fronteira (Argentina-Brasil-Paraguai). O trabalho privilegiou o diálogo entre a prática profissional e a formação acadêmica na modalidade de Residência Multiprofissional em Saúde da Família, buscando 
destacar as potencialidades da aprendizagem no trabalho através da perspectiva do cuidado integral à saúde nos diferentes ciclos da vida familiar. O sanitarista possui um perfil de atuação que visa o fortalecimento do Sistema Único de Saúde na atual conjuntura sociossanitária, estando altamente capacitado para lidar com questões relativas à vigilância em saúde, gestão das redes de cuidado, comunicação e educação em saúde e análise e planejamento situacional.

DESCRITORES: Capacitação de Recursos Humanos em Saúde. Saúde Coletiva. Infecções por Coronavírus.

\begin{abstract}
In view of the current socio-sanitarian crisis caused by the new manifestation of the coronavirus, this experience report sought to describe the insertion of hygienist in the Department of Epidemiological Surveillance in Foz do Iguaçu/PR, in the scope of the prevention and fight against Covid-19 in a Brazilian triple-border municipality. This work privileged the dialogue between professional practice and academic training in the modality of Multiprofessional Residency in Family Health, seeking to highlight the potential of learning at work through the perspective of comprehensive health care in the different cycles of family life. The hygienist has a profile of action that aims to strengthen the Unified Health System in the current socio-health situation, being highly qualified to deal with issues related to health surveillance, management of healthcare networks, health communication and education and analysis and situational planning tasks.
\end{abstract}

DESCRIPTORS: Training of Human Resources in Health. Collective Health. Coronavirus infections.

\title{
INTRODUÇÃO
}

A emergência sociossanitária mundial causada pela nova manifestação do coronavírus merece ser considerada como uma das epidemias mais significativas da história da humanidade. Numa visão sanitarista, pandemias são tidas como doenças infecciosas que se espalham por grandes regiões, ocorrendo ao redor do mundo praticamente ao mesmo tempo ${ }^{1}$, tendo como produto final o elevado coeficiente de morbimortalidade e letalidade; consequentemente, representando maiores desafios no âmbito social, econômico e político nos territórios acometidos. 
Informações recentes indicam que "a doença que o vírus produz é a COVID19 , onde 'CO' significa corona; 'Vl' vírus e 'D' doença”':1. Os tipos de coronavírus são agrupados em quatro (alfa; beta; delta; e gama); onde se tem registrado que somente o tipo alfa e beta infectam seres humanos, provocando, por sua vez, a síndrome respiratória aguda severa (SARS-CoV)². A referida síndrome "possui alto índice de mutação genética e fraca resistência a altas temperaturas $\left(56^{\circ} \mathrm{C}\right)$ por trinta minutos; etanol (75\%); desinfetantes que contenham cloro e ácido paracético"s:4031.

A Covid-19 tem sido descrita como uma doença com alta capacidade de infecção, porém com baixo coeficiente de letalidade ${ }^{1}$. O padrão de transmissão pode ocorrer em todas as faixas etárias da mesma forma, entretanto, o perfil epidemiológico mundial aponta para os idosos (pessoas com 60 ou mais anos de idade) e indivíduos com registro de comorbidades como principais grupos vulneráveis ${ }^{1-3}$.

Em 12 de janeiro de 2020, a Organização Mundial da Saúde (OMS) anunciou a Covid-19 como uma doença epidêmica, incentivando todas as organizações sanitárias, nacionais e internacionais a reforçar as medidas de prevenção, monitoramento e vigilância epidemiológica ${ }^{4}$. No Brasil, entretanto, intervenções formais foram decretadas pelo Ministério da Saúde em 3 de fevereiro de 2020, por meio da portaria $n^{\circ} 188$, a qual teve por definição declarar emergência em saúde pública de importância nacional (art. $1^{\circ}$ ) e estabelecer o Centro de Operações de Emergências em Saúde Pública (COE-nCoV) como mecanismo nacional da gestão coordenada da resposta à emergência no âmbito nacional (art. $\left.2^{\circ}\right)^{5}$.

A nível estadual, o Paraná decretou no Diário Oficial n 10.653, de 23 de março de 2020, estado de calamidade pública, seguindo orientações da Portaria $n^{\circ}$ $188^{6}$. Não obstante tal demora, a ativação do Centro de Operações em Emergências (COE) para o enfrentamento do novo coronavírus já tinha sido decretado mediante Resolução n 126/2020, da Secretaria de Estado da Saúde do Paraná (SESA/PR), sendo estabelecidas as estratégias e tipos de intervenções a serem realizadas no sistema de saúde paranaense?. Por outro lado, a Secretaria Muni- 
cipal da Saúde do município de Foz do Iguaçu/PR (SMSA/Foz), ciente das características dinâmicas de seu território, por se localizar em região de tríplice-fronteira (Argentina-Brasil-Paraguai), respondendo por importante demanda de serviços assistenciais para usuários estrangeiros e interestaduais, vem acompanhando o marco jurídico- sanitário tanto a nível nacional como estadual, com o objetivo primário de agir de forma consonante com as atividades desenvolvidas no território paranaense.

Torna-se evidente que a Covid-19 mostra como principal desafio para os sistemas de saúde e de assistência social a rápida capacidade de colonização e infecção do vírus, sendo mais rápida e eficaz do que as barreiras físicas e sociais adotadas pelos governos como medidas de controle para evitar a disseminação8. Dito desafio agrava ainda mais o quadro sociossanitário, ao se considerarem questões como: a alta demanda de atendimentos por suspeita; índice de admissão em unidade de terapia intensiva; custos por internação e intubação hospitalar; quantidade de leitos hospitalares disponíveis; organização das redes locais de assistência e alongadas jornadas de trabalho dos profissionais da saúde.

Entendendo a magnitude da situação sociossanitária que o Brasil enfrenta atualmente, em relação à prevenção e combate da Covid-19, traçou-se como objetivo geral de pesquisa destacar a participação do profissional sanitarista na rede local de saúde, ressaltando as aptidões e atribuições profissionais desenvolvidas no âmbito do Departamento de Vigilância Epidemiológica do município de Foz do Iguaçu/PR (DVE/Foz). Como objetivo conexo, este trabalho pondera o reconhecimento do sanitarista como profissional chave para a atenção primária no âmbito do Sistema Único de Saúde (SUS), na medida em que afirma a importância da sua presença e atuação não somente em tempos de crise epidêmica.

\section{MÉTODO}

Trata-se de um relato de experiência de percurso profissional, de cunho analítico-descritivo e com abordagem qualitativa, que aborda a inserção e atuação do 
sanitarista na rede local de saúde de Foz do Iguaçu/PR, no contexto de prevenção e combate da Covid-19.

Metodologicamente, os relatos de experiência indicam a observação, elaboração e expressão da experiência vivenciada na forma de uma narrativa9. Mais significativamente, narrar a experiência pretende, para mais de contar o vivido, fazer com que as informações relatadas prestem atenção às questões contemporâneas do campo científico atendido. Isto é, o relato de experiência é tido como uma ótima ferramenta para a atualização dos saberes e o aprimoramento das práticas em determinada área.

Tomou-se como cenário de práxis ao DVE/Foz. Cabe esclarecer que a vigilância epidemiológica é um conjunto de ações que proporcionam o conhecimento, detecção ou prevenção de qualquer mudança nos fatores determinantes e condicionantes de saúde individual e/ou coletiva, com a finalidade de recomendar e adotar as medidas de prevenção e controle dos agravos ${ }^{4}$. As atividades começaram a serem desempenhadas em 23/03/2020, estando atualmente em vigência (julho de 2020). Em relação à carga horária e vínculo de atuação profissional, os residentes possuem dedicação exclusiva, cumprindo uma carga horária semanal de 60 horas (de segundas-feiras a sábados).

Tratando as características do território de atuação, Foz do Iguaçu é um município trifronteiriço (Argentina-Brasil-Paraguai) localizado no extremo oeste paranaense, sendo categorizado pelo Instituto Brasileiro de Geografia e Estatística $(\mathrm{IGBE})^{10}$ como município de médio porte, com uma população estimada, até 2019, de 258.532 habitantes, com densidade demográfica correspondente a 418,58 habitantes $/ \mathrm{km}^{2}$.

A atuação dos sanitaristas no DVE/Foz está vinculada ao Programa de Residência Multiprofissional em Saúde da Família (PRMSF), da Universidade Federal da Integração Latino-Americana (UNILA), do qual os referidos profissionais fazem parte, na modalidade de aprendizagem no trabalho. Cumpre informar que, embora os profissionais possuam total autonomia, em relação às atribuições profissionais, a 
presença de um preceptor se faz necessária, segundo regulamento interno do PRMSF da UNILA, com o objetivo de fortalecer o processo de ensino- aprendizagem no local de trabalho. Nomeadamente, o PRMSF tem por objetivo principal "formar profissionais de saúde com conhecimentos, habilidades e atitudes para atuarem na Estratégia de Saúde da Família e Núcleo Ampliado de Saúde da Família, na perspectiva do cuidado integral à saúde nos diferentes ciclos de vida" familiar, considerando o planejamento estratégico local, a organização do processo de trabalho em equipe e as abordagens interdisciplinares e intersetoriais no campo da saúde ${ }^{11}$.

As atividades desenvolvidas pelos residentes sanitaristas foram agrupadas de forma descritiva e comparativa, isto é, o trabalho propõe destaque dos principais eixos de formação do sanitarista, logo complementadas por bibliografia da área. Estruturalmente, a discussão foi apresentada por meio de duas categorias: 1) formação acadêmica em saúde coletiva (permitindo entender algumas questões filosóficas da saúde coletiva, principalmente no que diz respeito à desconstrução do pensamento biomédico) e; 2) atuação do sanitarista no combate à Covid-19 no DVE/Foz (abrindo caminho para a compreensão do papel desse profissional na conjuntura da crise sanitária local).

Por se tratar de um relato de experiência de percurso profissional, o presente trabalho dispensou aprovação de Comitê de Ética em Pesquisa, segundo orientações da Resolução n 466 do Ministério da Saúde ${ }^{12}$.

\section{RESULTADO E DISCUSSÃO}

\section{Formação acadêmica em Saúde Coletiva}

Em um primeiro momento, faz-se jus levantar breves considerações em relação ao perfil profissional do sanitarista e sua inserção no sistema de saúde brasileiro. Assim, cabe entender que a Saúde Coletiva, como ciência própria, "vem a substituir a noção hegemônica que se tem sobre medicina social, ao mesmo tempo em que propõe uma nova estruturação da Saúde Pública brasileira"13:4. Desse modo, a 
Saúde Coletiva deve ser considerada como um projeto de construção de um saber que opera no campo da saúde, e também como um programa que contempla especificidade desse campo. Tal posicionamento denota a interdisciplinaridade que caracteriza a Saúde Coletiva como área contra hegemônica na saúde ${ }^{13}$.

O sanitarista é um profissional com formação acadêmica interdisciplinar na área da saúde humana, tendo como principais eixos de atuação: a gestão e planejamento em saúde; epidemiologia; comunicação e educação em saúde; e ciências sociais aplicadas à saúde. É, portanto, um profissional altamente capacitado para desenvolver suas atribuições em todos os níveis de atenção à saúde, embora sua presença no SUS prevaleça no nível primário.

Em visão ampla, em relação à formação e à atuação do sanitarista no contexto do SUS, cumpre dizer que:

A atual formação acadêmica do sanitarista parece, ainda, preservar e privilegiar algumas características da lógica da Saúde Pública. A organização pedagógica e curricular privilegia uma formação crítica, técnica e analítica, cuja ênfase recai: na operação com recursos estatísticos e epidemiológicos, análise de situações de saúde, e capacidade para refletir a partir das contribuições das ciências sociais e humanas, para operar com a análise de políticas e de práticas em saúde. Embora a diferenciação crítica e ideológica, a ênfase da formação do sanitarista moderno se aproxima de elementos que caracterizam o sanitarista Pré-Reforma Sanitária. No campo do mercado de trabalho, a expectativa dos serviços que acolhem o sanitarista também mantém, no imaginário, aquele profissional que sabia proceder e planejar ações no campo da gestão e da vigilância em saúde a partir da sua tradicional faceta epidemiológica. É possível que, atualmente, o próprio aspirante ao diploma dessa área profissional assuma, como importante para sua sobrevivência no mercado de trabalho, o desenvolvimento do saber-fazer tradicional da gestão e avaliação de políticas, além do valorizado conhecimento epidemiológico e estatístico ${ }^{14: 16 .}$

Por outro lado, a filosofia sanitarista banaliza a injustiça social, haja vistas dos Determinantes Sociais em Saúde, ora entendidos sob as lentes da subjetividade coletiva. Dita questão descreve subjetividade em saúde como "o produto 
de redes da história, descentrada do indivíduo, coletiva e nunca individual; é inventada e atende a interesses determinados, sendo produzida nos sentidos coletivos da sociedade"15:361. Para a Saúde Coletiva, o sujeito (usuário/cidadão) configura peça-chave no próprio desenvolvimento e aprimoramento do sistema e serviços de saúde, categorizando-o como um "agente de produção de discursos capazes de provocar mudanças no contingente homogeneizante instituídos pelos modelos de subjetivação capitalísticos"115:367.

Logo, a formação acadêmica em Saúde Coletiva contempla um conjunto de competências gerais e específicas: análise e monitoramento da situação de saúde; planejamento, programação, gestão e avaliação de sistemas e serviços de saúde; promoção da saúde e prevenção de riscos e agravos à saúde; gerenciamento de processos de trabalho coletivo em saúde; ética em Saúde Coletiva. Tais atividades são pensadas para serem desenvolvidas em todos os níveis da atenção à saúde, justamente porque o que se busca é a problematização das situações/condições de vida da sociedade (coletivo) numa visão integral, equitativa e universal ${ }^{16}$.

\section{Atuação do sanitarista no combate à Covid-19 no DVE/Foz}

No intuito de oferecer acompanhamento integral aos casos (suspeitos, confirmados e/ou descartados) e às diversas demandas vinculadas ao combate e controle da crise sanitária da Covid-19 no município de Foz do Iguaçu/PR, foi estabelecido o GT Covid/Foz, sendo um grupo de trabalho composto por uma equipe multiprofissional de servidores públicos e acadêmicos de nível superior (ora incluídos os sanitaristas residentes), todos com atuação na rede local de saúde iguaçuense.

No Quadro 1 são agrupadas e descritas as atividades desempenhadas pelos sanitaristas residentes no DVE/Foz. Percebe-se que as atividades são agrupadas em consonância com o eixo principal de formação do sanitarista. 
Quadro 1. Atividades desempenhadas pelos sanitaristas residentes segundo eixo de formação em Saúde Coletiva, DVE/Foz do Iguaçu, Paraná, 2020.

\begin{tabular}{|c|c|}
\hline Eixos formadores & Descrição das atividades \\
\hline \multirow{5}{*}{$\begin{array}{l}\text { GESTÃO E PLANEJAMENTO EM } \\
\text { SAÚDE - G\&PS }\end{array}$} & $\begin{array}{l}\text { *Reconhecimento do território, em } \\
\text { relação aos índices sanitários e } \\
\text { sociais (diagnóstico situacional de } \\
\text { saúde); }\end{array}$ \\
\hline & $\begin{array}{l}{ }^{*} \text { Capacitação para o manejo das } \\
\text { ferramentas institucionais (e-SUS } \\
\text { VE, REDCap, Consent, Notifica } \\
\text { Covid-19, SIVEP GRIPE, Sistema de } \\
\text { Informação de Agravos de Notificação } \\
\text { - SINAN, Gestor de Ambiente Laboral } \\
\text { - GAL, Base de dados institucional do } \\
\text { Covid-19); }\end{array}$ \\
\hline & $\begin{array}{l}{ }^{* * *} \text { Reformulação do fluxo de trabalho dos } \\
\text { profissionais membros do GT Covid/Foz } \\
\text { em relação à orientação das atividades a } \\
\text { serem realizadas, mudanças no processo } \\
\text { de trabalho, capacitações; }\end{array}$ \\
\hline & $\begin{array}{l}\text { ****Elaboração de relatórios e } \\
\text { arquivamento institucional de } \\
\text { informações relacionadas à gestão e } \\
\text { processo de trabalho do GT Covid- } \\
\text { Foz; }\end{array}$ \\
\hline & $\begin{array}{l}{ }^{* * * * *} \text { Elaboração dos certificados de } \\
\text { cumprimento do isolamento domiciliar } \\
\text { e de testagem não reagente. }\end{array}$ \\
\hline
\end{tabular}




\begin{tabular}{|c|c|}
\hline \multirow[t]{3}{*}{ EPIDEMIOLOGIA - EPI } & $\begin{array}{l}\text { *Recepção, revisão, correção e } \\
\text { tabulação dos dados registrados } \\
\text { nos prontuários (ficha de notificação } \\
\text { Covid-19), encaminhados pelos } \\
\text { diversos dispositivos sanitários, em } \\
\text { base de dados institucional. Em seguida, } \\
\text { notificação compulsória dos casos } \\
\text { nas correspondentes bases nacionais } \\
\text { (REDCap e Notifica Covid-19); }\end{array}$ \\
\hline & $\begin{array}{l}\text { **Análise estatística e epidemiológica } \\
\text { dos casos (em suspeita, confirmados e/ } \\
\text { ou descartados) registrados pelo DVE/ } \\
\text { Foz. Em seguida, georreferenciamento } \\
\text { dos pontos de concentração de contágio } \\
\text { da Covid-19 no município; }\end{array}$ \\
\hline & $\begin{array}{llr}{ }^{* * *} \text { Elaboração } & \text { dos } & \text { Boletins } \\
\text { Epidemiológicos } & \text { Covid/Foz a a } \\
\text { serem publicados pelo DVE/Foz, } \\
\text { segundo orientações técnicas da SESA/ } \\
\text { PR e SMSA/Foz; }\end{array}$ \\
\hline \multirow{4}{*}{$\begin{array}{l}\text { COMUNICAÇÃO E EDUCAÇÃO EM } \\
\text { SAÚDE - C\&ES }\end{array}$} & $\begin{array}{l}\text { *Acompanhamento longitudinal dos } \\
\text { casos (em suspeita, } \\
\text { confirmados e/ou descartados) } \\
\text { registrados pelo DVE/Foz por meio de } \\
\text { ferramentas de e-Health; }\end{array}$ \\
\hline & $\begin{array}{l}{ }^{* *} \text { Esclarecimento de dúvidas recebidas } \\
\text { nos diversos canais de informação } \\
\text { disponibilizados pelo DVE/Foz } \\
\text { em relação ao acompanhamento, } \\
\text { notificação e manejo dos casos } \\
\text { sint omáticos/assint o máticos } \\
\text { relacionados à Covid-19; }\end{array}$ \\
\hline & $\begin{array}{ll}{ }^{* * *} \text { Preenchimento dos } \mathrm{d} \text { a d } \mathrm{d} & \mathrm{s} \\
\text { ignorados } & \text { nas fichas } \mathrm{d} \text { e } \\
\text { notificação da COVID-19, com vistas a } \\
\text { manter o registro fiel } \\
\text { das informações; }\end{array}$ \\
\hline & $\begin{array}{l}{ }^{* * \star} \text { Educação popular com os usuários } \\
\text { por meio dos diversos canais de } \\
\text { informação, em relação às medidas de } \\
\text { prevenção e de isolamento domiciliar } \\
\text { dos casos em acompanhamento. }\end{array}$ \\
\hline
\end{tabular}

Fonte: Elaborado pelos autores (2020). 
No eixo da G\&PS sobressai a constante participação da preceptora, na modalidade de supervisão pedagógica. Assim, a função da preceptoria proposita o correto acompanhamento das normas sanitárias, uma vez que o posicionamento do SUS local se mantém em constante mudança, justificado pelo comportamento epidemiológico da Covid-19 no município. A fim de se familiarizar com os instrumentos de gestão e planejamento adotados pelo DVE/Foz no combate à Covid-19, foi realizada uma capacitação teórico-prática das ferramentas: e-SUS VE, Consent, SIVEP GRIPE, REDCap, Notifica Covid-19, SINAN e GAL.

Como ponto inicial no eixo G\&PS, teve-se a realização do diagnóstico situacional de saúde no contexto da crise sanitária da Covid-19. Importou entender as potencialidades do SUS local em relação à articulação das diversas tarefas de prevenção e cuidado. Especialmente, interessou entender as funções do DVE/Foz na perspectiva da intersetorialidade em saúde. Dessa forma, constatou-se o exposto na literatura ${ }^{17}$, ao afirmar que o processo de trabalho dos profissionais do SUS deve partir da compreensão holística dos problemas e necessidades de pessoas e grupos sociais em seus territórios. Partindo desse entendimento, foram estabelecidas prioridades para o manejo daqueles usuários considerados dentro do grupo de risco, sendo eles: idosos, crianças, grávidas, indivíduos com histórico de comorbidade e profissionais da saúde atuando na linha de frente.

A prática intersetorial com outras Secretarias Municipais se fez obrigatória na observância de contextos de vulnerabilidade social em usuários do grupo de risco. No caso dos idosos com diagnóstico positivo para Covid-19 e residindo sozinhos, foi ativada a Assistência Social do município, justamente pela situação de risco decorrente da doença. O acompanhamento do pré-natal foi mantido ainda nos casos de grávidas terem resultados reagentes à doença, considerado como serviço essencial para a saúde da mulher e da criança. Usuários com registro de comorbidade mereceram olhar diferenciado, por serem os indivíduos no grupo de risco com maior vulnerabilidade, em especial aqueles com patologias afetando aos sistemas respiratório e circulatório. Em ambos os casos, as atividades de G\&PS visaram estabelecer o vínculo ativo entre os usuários e as unidades de saúde. 
A atuação dos sanitaristas residentes no eixo EPI visou, em primeiro lugar, disponibilizar dados fiéis sobre o comportamento epidemiológico da Covid-19 em Foz do Iguaçu/PR. Dita função versa benefícios conexos, pois, por um lado, a análise da situação epidemiológica auxilia nas decisões institucionais do DVE/ Foz e, por outro, permite que a comunidade se mantenha informada, de forma atualizada, no que diz respeito aos coeficientes de mortalidade, contágio e ocupação dos leitos hospitalares. Entender o real alcance da Covid-19 no território iguaçuense se torna necessário quando se pensam, por exemplo, os mecanismos de prevenção e de assistência. Isto é, o comportamento epidemiológico da doença também sustenta o decreto de normas municipais mais estritas, a saber: o peneiramento de atividades comerciais, acadêmicas e a própria restruturação dos serviços locais de saúde, na tentativa de controlar a propagação do vírus.

Outrossim, a elaboração do Boletim Epidemiológico Covid-19/Foz requisita aptidões estatísticas já contempladas na formação do sanitarista e, no cenário atual, destaca-se ainda mais sua valia prática. Nesse sentido, a atuação no eixo EPI tem corroborado a afirmação de que "a epidemiologia é um dos pilares da Saúde Coletiva, devendo estar incorporada às políticas, programas e serviços de saúde"18:2. O desafio no eixo EPI, contudo, se evidenciou na qualidade do preenchimento das fichas de notificação encaminhadas ao DVE/Foz pelos diversos dispositivos sanitários (UBS, UPAS, Hospitais, Farmácias, entre outros). Ao longo das atividades, inúmeras notificações apresentaram inconsistência no grau de apuração fidedigna dos casos acompanhados, dificultando a qualidade e a real abrangência das informações oficiais da Covid-19 no DVE/Foz. De forma a atender tal entrave, de cunho técnico, diversas chamadas para a conscientização por parte dos profissionais encarregados da notificação dos casos foram promulgadas. Complementarmente, atividades no eixo C\&ES também mostraram resultados positivos para o eixo EPI, no que disse respeito à comprovação dos dados registrados nas fichas de notificação.

A compreensão dos determinantes sociais em saúde configura importante 
função para o profissional sanitarista. Entender os efeitos epidemiológicos da pandemia da Covid-19 requer, indubitavelmente, admitir com igual grau de importância os efeitos no tecido social e econômico observados no território paranaense. Isto porque a pandemia da Covid-19 tem desvelado os já conhecidos entraves dos sistemas de saúde e de assistência social no Paraná. Nesse sentido, o posicionamento do sanitarista diante de tal situação reforça a compreensão multidimensional da tríade saúde-doença-cuidados, numa visão biopsicossocial da saúde.

Nesse contexto sociossanitário, a vigilância do comportamento epidemiológico da Covid-19 fortalece as tarefas de identificação e acompanhamento dos casos, na medida em que permite obter informações chaves para repensar (em complemento ao eixo G\&PS) as intervenções sanitárias, principalmente no nível primário da atenção à saúde, sem, contudo, menosprezar o diálogo entre os outros níveis de atenção à saúde.

O eixo C\&ES, por sua vez, tem acenado tarefas de alto grau de complexidade. Primeiramente, ao rever a questão da longitudinalidade (continuidade do cuidado) em saúde, mesmo em tempos de crise sanitária, se torna importante "a presença do vínculo, relação interpessoal e de confiança entre usuários e profissionais de saúde"19:187. Ademais, a longitudinalidade "torna oportuno um cuidado integral, com ações de prevenção de doenças e promoção em saúde, e redução de uso de serviços de alta complexidade e custos na saúde"19:187.

Se bem, a atual reorganização dos serviços de saúde visualiza mecanismos orientados à contenção do coeficiente de contágio do vírus, na medida em que se tem limitado o fluxo de atendimento in loco, o SUS abraçou o desafio de levar o atendimento integral até a residência dos usuários. Para tanto, as intervenções adotaram a telessaúde como canal imediato de contato usuário-profissional-sistema de saúde, sendo essa uma ótima maneira para universalizar, integrar e fazer equitativo o cuidado à saúde, promovendo ao mesmo tempo o cumprimento das medidas de isolamento domiciliar e social. 
Por meio de telefonemas a linhas fixas e móveis, foi realizado o acompanhamento oportuno (de forma diária) dos casos acompanhados pelo GT Covid/ Foz no DVE/Foz. Importou registrar mudanças do quadro sintomatológico dos casos que receberam confirmação reagente para a Covid-19. Na ocasião, informações relativas às medidas de prevenção e de isolamento domiciliar eram retomadas com usuários, a fim de conscientizá-los sob a responsabilidade jurídica adquirida no ato de assinatura do termo de isolamento domiciliar. Eis que os telefonemas propiciaram espaço para o esclarecimento de dúvidas que emergiram ao longo do período de isolamento. Através das ligações, percebeu-se a importância da humanização dos cuidados oferecidos, embora essas não tenham tido a finalidade de medicar e/ou propor intervenções diretas no perfil de saúde dos usuários, não sendo essas atribuições profissionais do sanitarista.

Não obstante, nos casos onde identificada mudança negativa do quadro sintomatológico da Covid-19, isto é, uma piora no estado de saúde, os usuários eram orientados a entrarem em contato direto com as linhas de atendimento disponibilizadas pela SMSA/Foz (Plantão Coronavírus), do Hospital Municipal Padre Germano Lauck (HMPGL). Cabe informar que o HMPGL foi definido como o ponto de referência para a realização dos testes de biologia molecular (PCR). Dessa forma, ao ligarem para o Plantão Coronavírus, em jornada contínua 24h/7d, o usuário seria recepcionado por um acadêmico do curso de Medicina da UNILA, a fim de esclarecer as dúvidas médico-assistenciais e de prosseguir com o encaminhamento do paciente, segundo o grau de risco.

Uma questão amplamente notada e discutida, em relação ao eixo C\&ES, foi a necessidade de transmitir as informações por meio da linguagem coloquial (educação popular), pois houve, em muitas ocasiões, casos nos quais os usuários expressaram não entender as orientações repassadas pelos próprios profissionais da saúde. Isso foi evidenciado quando, por exemplo, os usuários eram questionados da existência de doenças crônicas (p.e. hipertensão arterial, para "pressão alta"; dispneia para "falta de ar" ou "dificuldade para respirar"; mialgia para "dor muscular", entre outras). Cumpre também lembrar que "a 
educação popular, como prática libertadora, é o instrumento pelo qual educador(a) e educando(a), tendo o mundo real como fonte de problematização, colocam a práxis humana em prática" 20:74.

A emissão das cartas de liberação do isolamento domiciliar ou de testagem não reagente para Covid-19 foi estruturada também por meio de estratégias do e- Health. Assim, foi criado um domínio de Gmail para recebimento desses pedidos, posteriormente endereçados com a correspondente liberação sanitária. Havendo registro de usuários sem acesso ao e-mail, estabeleceu um canal de comunicação pelo WhatsApp, mantendo a mesma lógica do Gmail, sendo as cartas de liberação encaminhadas no formato de arquivo portátil. Vê-se que tais estratégias de C\&ES obedeceram à necessidade de facilitar todas as formas possíveis para a continuidade do cuidado aos usuários acompanhados pelo GT Covid/Foz no DVE/Foz.

Finalmente, afirmamos que todos os eixos (G\&PS; EPI e C\&ES) possuem igual grau de incumbência nas atividades desempenhadas pelos sanitaristas residentes no DVE/Foz. A complementaridade entre ambos os eixos, isto é, a forma cíclica integrada, sugere a possibilidade de propor intervenções mais assertivas no âmbito do combate e prevenção da Covid-19 no território brasileiro de tríplice-fronteira. Tais atividades são essenciais para o ótimo acompanhamento epidemiológico e institucional da crise de Saúde Coletiva, pois permitem entender as características próprias da doença e seu comportamento, em relação à capacidade de resposta do SUS iguaçuense. A apropriação dos saberes interdisciplinares é, sem espaço de hesitação, importante elemento que auxilia ao sanitarista nas suas atribuições profissionais. 


\section{REFERÊNCIAS}

1. Tuñas ITC, Da Silva ET, Santiago SBS, Maia KD, Silva-Junior GO. Doença pelo coronavírus 2019 (COVID-19): uma abordagem preventiva para odontologia. [Internet]. Revista Brasileira de Odontologia; 2020 jan. 77(1):1-6. [citado em 2020 Jul 30]. Disponível em: http://dx.doi.org/10.18363/rbo.v77.2020.e1776.

2. Palacios MC, Santos E, Velázquez MAC, León J. COVID-19, una emergencia de salud pública mundial [Internet]. Revista Clínica Española; 2020 mar:1-7. [citado em 2020 Jul 30]. Disponível em: https://doi.org/10.1016/j.rce.2020.03.001.

3. Ahmad A, Rehman UM, Alkharfy KM. An alternative approach to minimize the risk of coronavirus (covid-19) and similar infections [Internet] European Review for Medical and Pharmacological Sciencies; 2020. (24):4030-4034. [citado em 2020 Jul 30]. Disponível em: 10.26355/eurrev_202004_20873.

4. Sun P, Lu X, Xu C, Sun W, Pan B. Understanding of COVID-19 based on current evidence [Internet]. Jornal of Medical Virology; 2020;1-4. [citado em 2020 Jul 30]. Disponível em: https://doi.org/10.1002/jmv.25722.

5. Brasil. Ministério da Saúde. Portaria $n^{\circ}$ 188, de 3 de fevereiro de 2020, declara emergência em saúde publica de importância nacional, em decorrência da infecção humana pelo novo coronavírus (COVID-19). [citado em 2020 Jul 30]. Disponível em: http://www.in.gov.br/en/web/dou/-/portaria-n-188-de-3-de-fevereiro-de-2020-241408388.

6. Paraná. Casa civil. Sistema Estadual de Legislação. Diário Oficial nº 10653, de 23 de março de 2020. Declara o estado de calamidade pública, para fins do art. 65, da Lei Complementar Federal n 101, de 4 de maio de 2000, com efeitos até 31 de dezembro de 2020, nos termos da solicitação do Governador do Estado, encaminhada à Assembleia Legislativa do Estado do Paraná, por meio da Mensagem no 15, de 23 de março de 2020. [citado em 2020 Jul 30]. Disponível em: https://leisestaduais.com.br/pr/decreto-n-4319-2020-parana-declara-o-estado-de-calamidade-publica-como-medida-paraenfrentamento-da-emergencia-de-saude-publica-de-importancia-internacional-decorrente-do-coronavirus-covid-19.

7. Paraná. Secretaria de Estado da Saúde do Paraná - SESA/PR. Resolução no 126/2020, ativar o Centro de Operações em Emergências - COE para o enfrentamento do novo coronavírus. Curitiba; 2020. [citado em 2020 Jul 30]. Disponível em: http://www.saude.pr.gov.br/sites/default/arquivos_restritos/files/documento/2020-04/126_20.pdf.

8. Marson FAL. Um milhão de casos de COVID-19: o que aprendemos? [Internet] São Paulo: Revista de Medicina; 2020, marabr;99(2):209-212. [citado em 2020 Jul 30]. Disponível em: https://doi.org/10.11606/issn.1679-9836.v99i2p209-212.

9. Domingo JC. Relatos de experiencia, en busca de un saber pedagógico [Internet]. Salvador: Revista Brasileira de Pesquisa (Auto) Biográfica; 2016, jan./abr;01(1):14-30. [citado em 2020 Jul 30]. Disponível em: http://dx.doi.org/10.31892/rbpab2525426X.2016.v1.n1.p14-30.

10. Instituto Brasileiro de Geografia e Estatística - IBGE. Cidades e Estados: Foz do Iguaçu. [citado em 2020 Jul 30]. Disponível em: https://www.ibge.gov.br/cidades-e-estados/pr/foz-do- iguacu.html.

11. Universidade Federal da Integração Latino-Americana - UNILA. Programa de Residência Multiprofissional em Saúde da Família. 2017. [citado em 2020 Jul 30]. Disponível em: https://portal.unila.edu.br/especializacao/residencia.

12. Brasil. Ministério da Saúde. Resolução no. 466, de 12 de dezembro de 2012. [citado em 2020 Jul 30]. Disponível em: http://conselho.saude.gov.br/resolucoes/2012/Reso466.pdf.

\section{RECEBIDO: $31 / 07 / 2020$ \\ ACEITO: $14 / 10 / 2020$}

